\title{
Diagnosis and management of protracted bacterial bronchitis: a survey of Chinese pediatricians
}

\author{
Qinyuan Li", , Ziyao Guo", Yuanyuan Li, Guangli Zhang, Xiaoyin Tian, Ruixue Gu, Zhengxiu Luo" \\ Department of Respiratory Medicine, Children's Hospital of Chongqing Medical University, National Clinical Research Center for Child Health \\ and Disorders, Ministry of Education Key Laboratory of Child Development and Disorders, Chongqing Key Laboratory of Pediatrics, Chongqing, \\ China \\ Contributions: (I) Conception and design: Z Luo, Q Li, Z Guo; (II) Administrative support: Z Luo; (III) Provision of study materials or patients: Z \\ Luo, Q Li, Z Guo, X Tian, R Gu; (IV) Collection and assembly of data: Q Li, Z Guo, Y Li, G Zhang; (V) Data analysis and interpretation: Q Li, Z \\ Guo; (VI) Manuscript writing: All authors; (VII) Final approval of manuscript: All authors. \\ \#These authors contributed equally to this work. \\ Correspondence to: Zhengxiu Luo, MD. Department of Respiratory Medicine, Children's Hospital of Chongqing Medical University, Chongqing, \\ China. Email: luozhengxiu816@163.com.
}

Background: Protracted bacterial bronchitis (PBB) is a common cause of chronic wet cough in children. However, it is often misdiagnosed and inappropriately treated in clinical practices. This study aims to investigate the current diagnosis and management of PBB among Chinese pediatricians.

Methods: An electronic questionnaire designed to assess the diagnosis and management of PBB was sent to pediatricians in China.

Results: A total of 1,022 pediatricians completed the questionnaire. Most (68.8\%) of the pediatricians diagnosed $\mathrm{PBB}$ in compliance with the guidelines, $44.3 \%$ and $24.5 \%$ of them followed microbiologybased and clinical-based diagnosis criteria, respectively. Only $40.4 \%$ of the pediatricians chose amoxicillinclavulanate as the first-line antibiotic for PBB treatment, $23.7 \%$ and $23.5 \%$ of them chose third-generation cephalosporins and macrolides, respectively. The majority of pediatricians (75.4\%) reported 2-4 weeks of antibiotics course, $19.3 \%$ of them prescribed a shorter course and $5 \%$ of them selected a longer course. Only $26.3 \%$ of the pediatricians performed combined investigations of chest high-resolution computed tomography scan (c-HRCT), bronchoscopy, and immunological tests for recurrences of PBB. Compared with general pediatricians (GP), pediatric pulmonologists (PP) preferred microbiology-based diagnosis criteria, prescribed more amoxicillin-clavulanate, and performed more investigations for recurrent patients $(\mathrm{P}<0.05)$.

Conclusions: The majority of Chinese pediatricians diagnosed PBB in compliance with guidelines. However, the reasonable antibiotics applications and the investigations for recurrent PBB need to be improved.

Keywords: Children; diagnosis; management; pediatrician; protracted bacterial bronchitis (PBB)

Submitted May 15, 2020. Accepted for publication Sep 24, 2020.

doi: $10.21037 / \mathrm{atm}-20-3984$

View this article at: http://dx.doi.org/10.21037/atm-20-3984

$\wedge$ ORCID: 0000-0002-4052-5747. 


\section{Introduction}

Chronic cough, defined as cough duration $>4$ weeks, is a common symptom in children (1). It causes high burden of recurrent medical visits (2) and impairs life quality of the children and parents $(1,3)$. A study found that more than $80 \%$ of chronic cough children sought $\geq 5$ medical consultations and $53 \%$ had $>10$ visits within 12 months (2). Protracted bacterial bronchitis (PBB) is one of the most common causes of chronic wet cough in children, exceeding by two-to-three fold other underlying diseases, including asthma $(1,4,5)$. PBB is associated with bacterial infection and inflammation of the bronchus $(6,7)$. Symptoms could be resolved following appropriate antibiotic treatment (8). However, PBB is frequently underdiagnosed and misdiagnosed as lack of specific manifestations (1). Some undiagnosed patients with inappropriate management may progress to chronic suppurative lung disease (CSLD) and/or bronchiectasis (7). Therefore, it is important to diagnose and manage PBB appropriately.

Guidelines are important tools in standardizing and improving clinical practice. The first guideline on the diagnosis and treatment of PBB in children was proposed in 2006 (9) and has been updated in recent years (10-12). Whether pediatricians implement these guidelines in clinical practice remains unclear. We conducted a survey-based study among Chinese pediatricians (I) to investigate their diagnosis and management of $\mathrm{PBB}$, (II) to compare practice differences towards $\mathrm{PBB}$ between general pediatricians (GP) and pediatric pulmonologists (PP), and (III) to assess the PBB guidelines compliance. We present the following article in accordance with the Survey Reporting Guideline (SURGE) reporting checklist (13) (available at http://dx.doi.org/10.21037/atm-20-3984).

\section{Methods}

\section{Study design}

This was a cross-sectional survey conducted from March 15 to 22, 2020 in China. We designed the questionnaire based on the guideline (14). Investigators designed the questionnaire based on the American College of Chest Physicians (CHEST) guidelines and European Respiratory Society (ERS) statement (10-12), as no validated and published questionnaires were available for reference. Three PP who specialized in PBB management, and one statistician specialized in survey research were consulted to improve the quality of the questionnaire. The questionnaire was pre-tested on a sample of 20 pediatricians, 5 of them worked in tertiary general hospitals, 5 in tertiary children's hospitals, 5 in secondary hospitals, and 5 in primary hospitals. The questionnaire was revised based on the comments provided by the specialists and the participants.

The survey was composed of an introductory letter and 8 questions on one page. The introductory letter contained information about the investigators and the purpose of the study. The questions aimed at obtaining the following: (I) demographic characteristics of pediatricians (professional title, specialty, workplace and confirmed cases of $\mathrm{PBB}$ per month); (II) the criteria of PBB diagnosis; (III) the preferred antibiotic for PBB; (IV) the antibiotic course; (V) the investigations for recurrent PBB patients. We thanked participants for their cooperation at the end of the questionnaire.

A questionnaire link was sent to Chinese pediatricians and further forwarded to their colleagues using the WenJuanXing web-application (web link: www.wjx.cn) via social media. After all questions were completed, the questionnaire could be submitted. Participants were unable to modify their answers after submission. All answers remained anonymous. No incentives were offered to obtain the survey results. Personal information of the participants was kept confidential and stored at the password-protected survey database. The study was conducted in accordance with the Declaration of Helsinki (as revised in 2013). The Ethical Committee of the Children`s Hospital of Chongqing Medical University approved the study protocol and the final questionnaire (No. 2020-53: the registration number of ethics board) and individual consent for this cross-sectional survey was waived. No conflicts existed in this survey.

\section{Definition}

The clinical-based diagnosis of PBB referred to the following criteria (10-12). (I) Presence of chronic wet cough; (II) absence of an alternative specific etiology; and (III) cough resolved following 2 weeks of appropriate oral antibiotic therapy. Microbiology-based diagnosis criteria of $\mathrm{PBB}$ were defined as follows (10-12): (I) presence of chronic wet cough; (II) identifiable respiratory bacterial pathogens growing in sputum or bronchoalveolar lavage (BAL) at a density of a single bacterial species $>10^{4} \mathrm{CFU} / \mathrm{mL}$; and (III) resolution of cough within 2 weeks of appropriate oral antibiotic. Recurrent PBB was defined as $\geq 3$ episodes of PBB per year. 
Table 1 Demographic characteristics of participants and compared between GP and PP, n (\%)

\begin{tabular}{|c|c|c|c|c|}
\hline Variables & Total $(n=1,022)$ & GP $(n=895)$ & $P P(n=127)$ & $P$ value \\
\hline Chief & $132(12.9)$ & $91(10.2)$ & $41(32.3)$ & $<0.05$ \\
\hline Associate chief & $282(27.6)$ & $246(27.5)$ & $36(28.3)$ & 0.839 \\
\hline Attending & $350(34.2)$ & $318(35.5)$ & $32(25.2)$ & $<0.05$ \\
\hline \multicolumn{5}{|l|}{ Hospital level } \\
\hline Tertiary general hospital & $412(40.3)$ & $350(39.1)$ & $62(48.8)$ & $<0.05$ \\
\hline Tertiary children's hospital & $159(15.6)$ & $102(11.4)$ & $57(44.9)$ & $<0.05$ \\
\hline Secondary hospital & $409(40)$ & $401(44.8)$ & $8(6.3)$ & $<0.05$ \\
\hline \multicolumn{5}{|c|}{ Confirmed PBB cases per month } \\
\hline 0 & $213(20.8)$ & $191(21.3)$ & $22(17.3)$ & 0.297 \\
\hline $1-5$ & $610(59.7)$ & $532(59.4)$ & $78(61.4)$ & 0.671 \\
\hline $5-10$ & $122(11.9)$ & $109(12.2)$ & $13(10.2)$ & 0.527 \\
\hline $10-15$ & $32(3.1)$ & $26(2.9)$ & $6(4.7)$ & 0.407 \\
\hline$\geq 15$ & $45(4.4)$ & $37(4.1)$ & $8(6.3)$ & 0.266 \\
\hline
\end{tabular}

GP, general pediatricians; PP, pediatric pulmonologists.

\section{Statistical analysis}

Data were analyzed using the SPSS Statistics 22.0 program (SPSS Inc, Chicago, IL, USA). Categorical data were presented as numbers (\%). Pearson Chi-square or Fisher's exact test was used to compare data between PP and GP. Statistical significance was considered at $\mathrm{P}<0.05$.

\section{Results}

\section{Demographic characteristics of participants}

A total of 1,022 Chinese pediatricians completed the questionnaire, $895(87.6 \%)$ of whom were GP and 127 (12.4\%) were PP. Nearly $80 \%$ of them worked in western China. Most pediatricians worked in tertiary general hospitals $(40.3 \%)$ and secondary hospitals (40\%). Over half of them $(59.7 \%)$ diagnosed $1-5$ cases of PBB per month. More PP were chiefs and worked in tertiary children's hospitals, compared with GP $(\mathrm{P}<0.05)$, as shown in Table 1 .

\section{Diagnosis of PBB}

Our survey showed that $68.8 \%(703 / 1,022)$ of the pediatricians diagnosed $\mathrm{PBB}$ in compliance with the published guidelines. A total of $44.3 \%(453 / 1,022)$ of the participants diagnosed PBB according to microbiologybased criteria and $24.5 \%(250 / 1,022)$ of them followed clinical-based diagnosis criteria. The guidelines compliance rate was significantly higher among PP than that in GP ( $83.5 \%$ vs. $66.7 \%, \mathrm{P}<0.05$ ). PP were more likely to diagnose $\mathrm{PBB}$ according to microbiology-based criteria when compared to GP ( $63 \%$ vs. $41.7 \%, \mathrm{P}<0.05)$. The compliance rate of clinical-based diagnosis criteria was similar between GP and PP (25\% vs. $20.5 \%, \mathrm{P}=0.264)$ (Figure 1).

\section{Therapy of PBB}

\section{Type of antibiotic}

Guidelines recommend oral amoxicillin-clavulanate for PBB children (10-12). Our survey indicated that $40.4 \%$ 


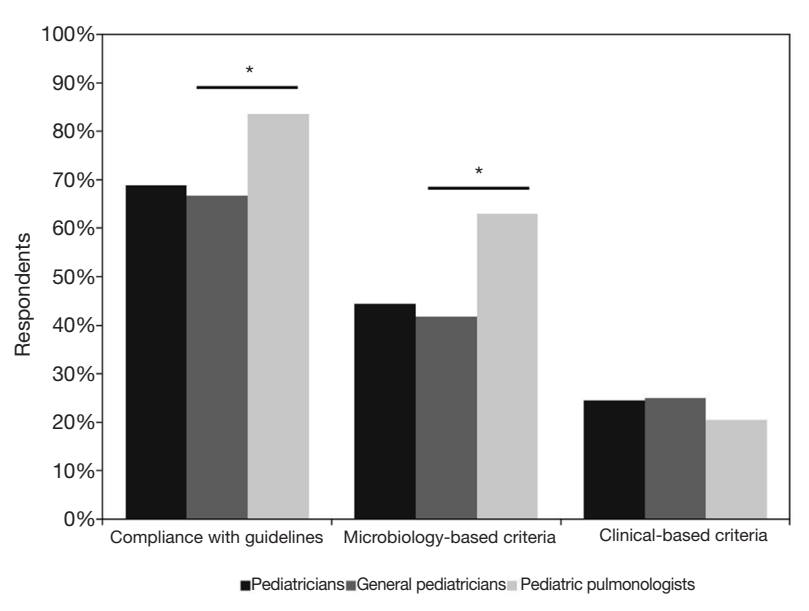

Figure 1 Criteria used in the diagnosis of PBB and compliance with the guidelines. *, general pediatricians compared with pediatric pulmonologists, $\mathrm{P}<0.05$. $\mathrm{PBB}$, protracted bacterial bronchitis.

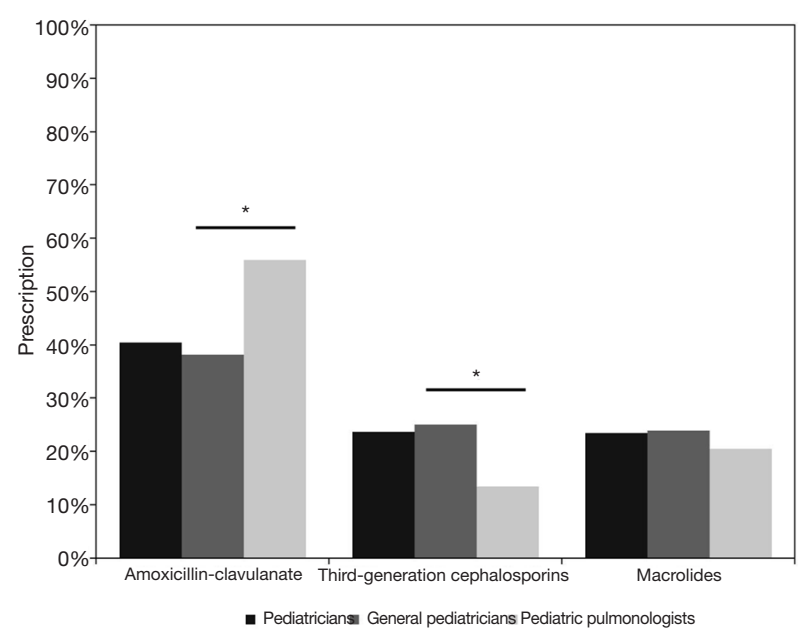

Figure 2 The three most common oral antibiotics prescribed for PBB patients. *, general pediatricians compared with pediatric pulmonologists, $\mathrm{P}<0.05$. $\mathrm{PBB}$, protracted bacterial bronchitis.

$(413 / 1,022)$ of the pediatricians preferred amoxicillinclavulanate in clinical practice. PP prescribed oral amoxicillin-clavulanate more frequently than GP $(55.9 \%$ vs. $38.2 \%, \mathrm{P}<0.05)$. Approximately a quarter $(23.7 \%$, $242 / 1,022)$ of participants chose oral third-generation cephalosporins. GP were more likely to select oral thirdgeneration cephalosporins when compared to PP $(25.1 \%$ vs. $13.4 \%, \mathrm{P}<0.05)$ (Figure 2). A total of $240(23.5 \%)$ pediatricians selected oral macrolides, with no significant

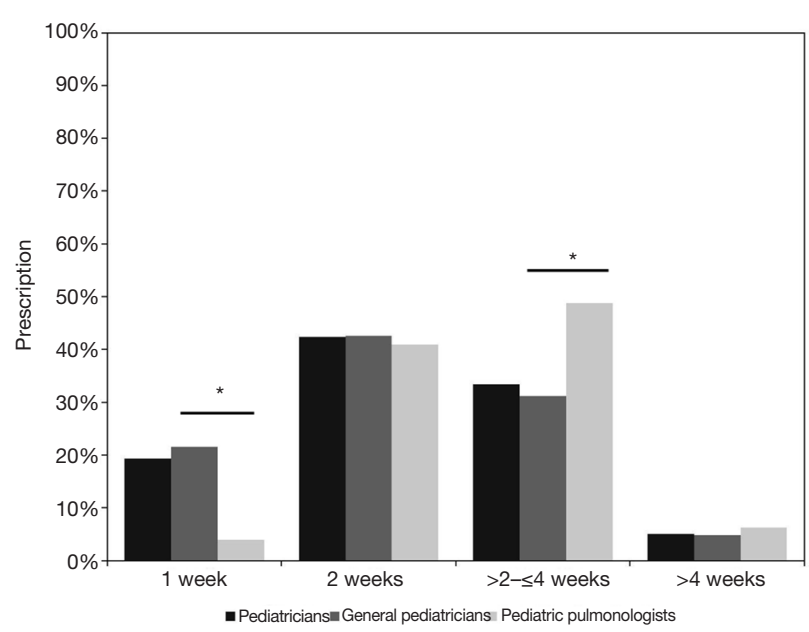

Figure 3 Duration of antibiotics for treatment of PBB. *, general pediatricians compared with pediatric pulmonologists, $\mathrm{P}<0.05$. $\mathrm{PBB}$, protracted bacterial bronchitis.

difference between PP and GP (20.5\% vs. $23.9 \%, \mathrm{P}=0.392)$. A total of $7.1 \%, 2.3 \%, 1.6 \%$ and $1.5 \%$ of the pediatricians chose second-generation cephalosporins, amoxicillin, firstgeneration cephalosporins, and other antibiotics, respectively. No significant difference was found in prescription of second-generation cephalosporins $(7.4 \%$ vs. $5.5 \%, \mathrm{P}=0.446)$, amoxicillin $(2.5 \%$ vs. $0.8 \%, \mathrm{P}=0.385)$, first-generation cephalosporins ( $1.7 \%$ vs. $0.8 \%, \mathrm{P}=0.45)$ and other antibiotics (1.2\% vs. $3.1 \%, \mathrm{P}=0.092)$ between GP and PP.

\section{Course of antibiotic therapy}

According to the guidelines, a 2-week course is sufficient for the majority of $\mathrm{PBB}$ patients, while 4 weeks may be required in some PBB patients (10-12). A total of 433 (42.4\%) pediatricians reported a 2 -week course, with no significant difference between PP and GP (40.9\% vs. $42.6 \%, \mathrm{P}=0.729$ ). Approximately $33 \%$ of the pediatricians (341/1022) indicated 2-4 weeks of an antibiotics course. PP were more likely to choose $2-4$ weeks than GP (48.8\% vs. $31.2 \%, \mathrm{P}<0.05$ ). A shorter duration (1 week) was chosen by $19.3 \%$ of the pediatricians and GP chose a 1-week duration more frequently than $\mathrm{PP}(21.5 \%$ vs. $3.9 \%, \mathrm{P}<0.05)$. We found $51(5 \%)$ pediatricians reported a longer course of more than 4 weeks, with no significant difference between $\mathrm{PP}$ and GP (6.3\% vs. 4.8\%, $\mathrm{P}=0.469)$, as shown in Figure 3.

\section{Investigations for recurrent $P B B$ patients}

Guidelines suggest patients with recurrent PBB should be 
Table 2 Investigations for patients with recurrent PBB, n (\%)

\begin{tabular}{|c|c|c|c|c|}
\hline Variables & Total $(n=1,022)$ & GP $(n=895)$ & $P P(n=127)$ & $P$ value \\
\hline Bronchoscopy with BAL & $621(60.8)$ & $513(57.3)$ & $108(85.0)$ & $<0.05$ \\
\hline Immunological tests & $453(44.3)$ & $371(41.5)$ & $82(64.6)$ & $<0.05$ \\
\hline nFeNO & $230(22.5)$ & $178(19.9)$ & $52(40.9)$ & $<0.05$ \\
\hline c-HRCT + bronchoscopy with BAL + immunological tests & $269(26.3)$ & $199(22.2)$ & $70(55.1)$ & $<0.05$ \\
\hline
\end{tabular}

GP, general pediatricians; PP, pediatric pulmonologists; PBB, protracted bacterial bronchitis; c-HRCT, chest high resolution computerized tomography; BAL, bronchoalveolar lavage; $\mathrm{nFeNO}$, exhaled fractional nasal nitric oxide.

further investigated for underlying diseases $(11,12)$. Further investigations should include at least chest high-resolution computed tomography (c-HRCT), bronchoscopy with BAL and immunological tests (15). A total of $67.4 \%$ of the pediatricians initiated a c-HRCT scan when PBB recurred. Bronchoscopy with bronchoalveolar lavage (BAL) was selected by $60 \%$ of pediatricians, followed by immunological tests $(44.3 \%)$, exhaled fractional nasal nitric oxide (nFeNO) (22.5\%) and genetic testing (18.8\%). Nearly a quarter of the participants $(26.3 \%)$ underwent c-HRCT, bronchoscopy with BAL, and immunological tests simultaneously. PP were more likely than GP to perform all of the three investigations ( $55.1 \%$ vs. $22.2 \%, \mathrm{P}<0.05)$, as shown in Table 2.

\section{Discussion}

PBB is a common cause of chronic wet cough in children. However, it is often misdiagnosed and inappropriately treated. Undiagnosed and inappropriately treated PBB may progress to CSLD or bronchiectasis, with life quality impaired. Guidelines have been developed to standardize and improve the management of PBB. However, the implementation of these guidelines in daily general practice remains unclear. In this study, we demonstrated that most Chinese pediatricians diagnosed PBB in compliance with guidelines. However, the reasonable antibiotic applications and the investigations for recurrent $\mathrm{PBB}$ need to be improved.

Guidelines recommend the diagnosis of PBB following clinical-based or microbiology-based criteria (10-12). In this survey, we found pediatricians preferred microbiologybased criteria. Positive BAL fluid (BALF) culture for bacteria is strong evidence for PBB diagnosis. However, antibiotic use before culture can induce false-negative results. Overemphasis of the positive results in BALF culture may lead to missed diagnosis of PBB. Moreover, bronchoscopy and BAL may be unfeasible in some pediatric clinics, especially in the primary centers and rural areas. It is unrealistic and unethical to undertake invasive bronchoscopy for every child with chronic wet cough. Therefore, it may be more appropriate to diagnose $\mathrm{PBB}$ according to clinicalbased criteria, especially for GP. Nevertheless, PP should perform bronchoscopy and BAL for patients with treatment failure before making PBB diagnosis. When bronchoscopy is performed, purulent secretions and evidence of bronchitis are usually present (16-18).

Haemophilus influenzae, Streptococcus pneumoniae, and Moraxella catarrbalis are the three most common pathogens of PBB (10-12). Guidelines recommend PBB patients receive antibiotics active against these bacteria (10-12). Antibiotic prescribing is usually empirical and determined by local patterns of antibiotic susceptibility. Amoxicillinclavulanate is recommended as the first-line antibiotic in empiric treatment to PBB $(10-12,16)$. Multicenter studies of antibiotic susceptibility in China showed that the susceptibility to amoxicillin-clavulanate among the three pathogens was high $(19,20)$, ranging from $75-100 \%$. This survey showed amoxicillin-clavulanate was the most commonly used antibiotic. However, the prescription rate of amoxicillin-clavulanate was only $40 \%$, which was far lower than the data reported by other studies (21-24). Therefore, more training and education are needed to increase rational prescription of amoxicillin-clavulanate. Thirdgeneration cephalosporins were the second most commonly used antibiotics, which were more frequently prescribed by GP. According to the guidelines, third-generation cephalosporins may be used as alternatives for patients with 
a history of immediate hypersensitivity to penicillin (10-12). Overuse of third-generation cephalosporins may increase the risk of bacterial resistance and adverse drug events $(19,25)$. More rational and restrained use of these agents should be emphasized. This survey showed that a quarter of the pediatricians chose macrolides as their preferred drugs. Azithromycin may be an effective treatment for CSLD or bronchiectasis (26). However, the role of macrolides in PBB remains undefined. Moreover, these pathogens associated with PBB were resistant to macrolides in China, especially Streptococcus pneumoniae with a high resistance rate of over $90 \%(19,20,27)$. Therefore, empirical macrolide therapy is not recommended for PBB patients.

An adequate course of antibiotics treatment is important for the prognosis of PBB. Guidelines recommend the 2 -week course of oral antibiotics for suspected PBB patients (10-12). Up to a 4-week duration is recommended for children with persistent wet cough after 2 weeks of antibiotics (10-12). This survey found most pediatricians followed guidelines for antibiotics courses. Some pediatricians used a shorter course and GP accounted for the majority. Inadequate course may cause cough recurrences and impair life quality (7). Therefore, education about antibiotic courses is warranted for pediatricians especially GP. A study indicated that PBB patients with no response to 4 weeks of antibiotics had a high risk of developing bronchiectasis (28). Whether the antibiotic course should be extended to more than 4 weeks remains unknown. Two studies found $25-51 \%$ of PBB patients were symptom free following 6-8 weeks of antibiotic treatment, while the others who received more courses of antibiotics or long-term prophylactic antibiotics still suffered recurrent episodes $(22,23)$. One latest study reported patients who received 6-week of antibiotics were less likely to develop recurrent PBB than those who received 2 weeks (29). Few pediatricians in our study chose the prolonged antibiotic course. However, the prognosis of recurrent PBB patients was unknown. Prospective studies of antibiotics course effects on PBB prognosis are further needed.

Investigations for underlying disease associated with PBB should be performed carefully (10-12). Evidence suggests $\mathrm{PBB}, \mathrm{CSLD}$, and bronchiectasis are related disease and aggravate gradually (16). Recurrent $\mathrm{PBB}$ is an independent risk factor for development of CSLD or bronchiectasis (30). Therefore c-HRCT is recommended for any child with recurrent PBB, especially if accompanied by symptoms and/or signs suggestive of bronchiectasis (15). Further investigations for other possible causes should be undertaken. The ERS task force stated that recurrences of PBB may be associated with airway malacia and/or immune deficiencies (11). Bronchoscopy with BAL and immunological tests may be required for suspected patients. Although c-HRCT, bronchoscopy, and immunological tests were the top three investigations performed by pediatricians, only a quarter of them chose all three investigations in our survey. These findings indicated that the pediatricians may lack comprehensive knowledge of the etiologies of recurrent PBB. It might be associated with little evidence to inform the optimal investigations. Existing guidelines do not provide a specific list of investigations for recurrent PBB. Besides, few studies have revealed the underlying diseases of recurrent $\mathrm{PBB}$. Therefore, more prospective studies should be conducted to reveal the underlying diseases of recurrent PBB.

To our knowledge, this is the first survey to investigate the current diagnosis and management of PBB among Chinese pediatricians. The results of this survey may be helpful for future pediatricians' educations and studies. However, there were some limitations. First, the selfreport results may be unequal to clinical practice. We tried to avoid this bias by conducting surveys in complete anonymity conditions. The results may be overestimated as the respondents may be prone to provide ideal answers. Further clinical studies are needed to clarify the actual clinical behavior of pediatricians. Second, most participants were from western China with small sample sizes, thus the findings may not be extrapolated to pediatricians in other areas. Third, the practices of the respondents may be different from those with no response, which may affect the validity of the survey. Fourth, we used a self-designed questionnaire as no validated and published questionnaires were available for reference. Nevertheless, we tried to improve the quality of this questionnaire by consulting specialists and pre-testing.

\section{Conclusions}

The majority of Chinese pediatricians diagnosed PBB in compliance with the guidelines. However, the reasonable antibiotic applications and the investigations for recurrent PBB need to be improved.

\section{Acknowledgments}

We thank pediatricians who kindly agreed to complete the survey. We thank experts for their review and supervision 
of the survey. We thank pediatricians who participated in the pre-testing and provided assistance in optimizing the questionnaire.

Funding: This work was supported by the Science and Technology Department of Chongqing (cstc2018jscxmsybX0021 to Zhengxiu Luo).

\section{Footnote}

Reporting Checklist: The authors have completed the SURGE reporting checklist. Available at http://dx.doi. org/10.21037/atm-20-3984

Data Sharing Statement: Available at http://dx.doi. org/10.21037/atm-20-3984

Conflicts of Interest: All authors have completed the ICMJE uniform disclosure form (available at http://dx.doi. org/10.21037//atm-20-3984). The authors have no conflicts of interest to declare.

Ethical Statement: The authors are accountable for all aspects of the work in ensuring that questions related to the accuracy or integrity of any part of the work are appropriately investigated and resolved. The study was conducted in accordance with the Declaration of Helsinki (as revised in 2013). The study was approved by the Ethical Committee of the Children's hospital of Chongqing Medical University (NO. 2020-53: the registration number of ethics board) and individual consent for this crosssectional survey was waived.

Open Access Statement: This is an Open Access article distributed in accordance with the Creative Commons Attribution-NonCommercial-NoDerivs 4.0 International License (CC BY-NC-ND 4.0), which permits the noncommercial replication and distribution of the article with the strict proviso that no changes or edits are made and the original work is properly cited (including links to both the formal publication through the relevant DOI and the license). See: https://creativecommons.org/licenses/by-nc-nd/4.0/.

\section{References}

1. Chang AB, Robertson CF, van Asperen PP, et al. A multicenter study on chronic cough in children: burden and etiologies based on a standardized management pathway. Chest 2012;142:943-50.
2. Marchant JM, Newcombe PA, Juniper EF, et al. What is the burden of chronic cough for families? Chest 2008;134:303-9.

3. Newcombe PA, Sheffield JK, Petsky HL, et al. A child chronic cough-specific quality of life measure: development and validation. Thorax 2016;71:695-700.

4. Asilsoy S, Bayram E, Agin H, et al. Evaluation of chronic cough in children. Chest 2008;134:1122-8.

5. Usta Guc B, Asilsoy S, Durmaz C. The assessment and management of chronic cough in children according to the British Thoracic Society guidelines: descriptive, prospective, clinical trial. Clin Respir J 2014;8:330-7.

6. Taussig LM, Smith SM, Blumenfeld R. Chronic bronchitis in childhood: what is it? Pediatrics 1981;67:1-5.

7. Chang AB, Redding GJ, Everard ML. Chronic wet cough: protracted bronchitis, chronic suppurative lung disease and bronchiectasis. Pediatr Pulmonol 2008;43:519-31.

8. Marchant JM, Morris P, Gaffney J, et al. Antibiotics for prolonged moist cough in children. Cochrane Database Syst Rev 2005;19:CD004822.

9. Chang $\mathrm{AB}$, Glomb WB. Guidelines for evaluating chronic cough in pediatrics: ACCP evidence-based clinical practice guidelines. Chest 2006;129:260S-83S.

10. Chang AB, Oppenheimer JJ, Weinberger MM, et al. Management of Children With Chronic Wet Cough and Protracted Bacterial Bronchitis: CHEST Guideline and Expert Panel Report. Chest 2017;151:884-90.

11. Kantar A, Chang AB, Shields MD, et al. ERS statement on protracted bacterial bronchitis in children. Eur Respir J 2017;50:1602139.

12. Chang AB, Oppenheimer JJ, Irwin RS, et al. Managing Chronic Cough as a Symptom in Children and Management Algorithms: CHEST Guideline and Expert Panel Report. Chest 2020;158:303-29.

13. Grimshaw, Jeremy. 20. SURGE (The SUrvey Reporting GuidelinE). Guidelines for Reporting Health Research: A User's Manual. John Wiley and Sons, Ltd., 2014.

14. Boynton PM, Greenhalgh T. Selecting, designing, and developing your questionnaire. BMJ 2004;328:1312-5.

15. Chang AB, Bell SC, Torzillo PJ, et al. Chronic suppurative lung disease and bronchiectasis in children and adults in Australia and New Zealand: Thoracic Society of Australia and New Zealand guidelines. Med J Aust 2015;202:21-3.

16. Chang AB, Upham JW, Masters IB, et al. Protracted bacterial bronchitis: The last decade and the road ahead. Pediatr Pulmonol 2016;51:225-42.

17. Narang R, Bakewell K, Peach J, et al. Bacterial distribution in the lungs of children with protracted bacterial 
bronchitis. PLoS One 2014;9:e108523.

18. Donnelly D, Critchlow A, Everard ML. Outcomes in children treated for persistent bacterial bronchitis. Thorax 2007;62:80-4.

19. Hu F, Zhu D, Wang F, et al. Results from the Survey of Antibiotic Resistance (SOAR) 2009-11 and 2013-14 in China. J Antimicrob Chemother 2016;71 Suppl 1:i33-43.

20. Hu F, Guo Y, Zhu DM, et al. CHINET surveillance of bacterial resistance in China: 2018 report. Chin J Infect Chemother 2020;20:1-10.

21. Marchant J, Masters IB, Champion A, et al. Randomised controlled trial of amoxycillin-clavulanate in children with chronic wet cough. Thorax 2012;67:689-93.

22. Donnelly D, Critchlow A, Everard ML. Outcomes in children treated for persistent bacterial bronchitis. Thorax 2007;62:80-4.

23. Pritchard MG, Lenney W, Gilchrist FJ. Outcomes in children with protracted bacterial bronchitis confirmed by bronchoscopy. Arch Dis Child 2015;100:112.

24. Kompare M, Weinberger M. Protracted bacterial bronchitis in young children: association with airway malacia. J Pediatr 2012;160:88-92.

25. Ren X, Liu D, Ding N, et al. Safety evaluation of cephalosporins based on utilization and adverse drug

Cite this article as: Li Q, Guo Z, Li Y, Zhang G, Tian X, Gu R, Luo Z. Diagnosis and management of protracted bacterial bronchitis: a survey of Chinese pediatricians. Ann Transl Med 2021;9(1):46. doi: 10.21037/atm-20-3984 events: analysis of two databases in China. Expert Opin Drug Saf 2012;11:689-97.

26. Valery PC, Morris PS, Byrnes CA, et al. Long-term azithromycin for Indigenous children with non-cysticfibrosis bronchiectasis or chronic suppurative lung disease (Bronchiectasis Intervention Study): a multicentre, double-blind, randomised controlled trial. Lancet Respir Med 2013;1:610-20. Erratum in: Lancet Respir Med 2015;3:e29.

27. Shi W, Wen D, Chen C, et al. $\beta$-Lactamase production and antibiotic susceptibility pattern of Moraxella catarrhalis isolates collected from two county hospitals in China. BMC Microbiol 2018;18:77.

28. Goyal V, Grimwood K, Marchant JM, et al. Does failed chronic wet cough response to antibiotics predict bronchiectasis? Arch Dis Child 2014;99:522-5.

29. Gross-Hodge E, Carroll WD, Rainford N, et al. Duration of initial antibiotic course is associated with recurrent relapse in protracted bacterial bronchitis. Arch Dis Child 2020;105:1111-3.

30. Wurzel DF, Marchant JM, Yerkovich ST, et al. Protracted bacterial bronchitis in children: natural history and risk factors for bronchiectasis. Chest 2016;150:1101-8. 